\title{
Adjuvant Therapy Clinical Trial Setting
}

National Cancer Institute

\section{Source}

National Cancer Institute. Adjuvant Therapy Clinical Trial Setting. NCI Thesaurus. Code

C124450.

A clinical trial or treatment setting in which the therapy of interest is considered adjuvant. 